\title{
Realistic medium-averaging in radiative energy loss
}

\author{
Denes Molnar, Deke Sun
}

Physics Department, Purdue University, West Lafayette, IN 47907

\begin{abstract}
We present results from a jet energy loss calculation using the Gyulassy-Levai-Vitev (GLV) formalism and bulk medium evolution from the covariant transport MPC. At both RHIC and LHC energies we find that realistic transverse expansion strongly reduces elliptic flow at high $p_{T}$ compared to calculations with transversely "frozen" profiles. We argue that this is a generic feature of GLV energy loss. Transverse expansion also leads to stronger high- $p_{T}$ suppression, while fluctuations in energy loss with the location of scattering centers weaken the suppression. But, unlike the reduction of $v_{2}$, these effects nearly disappear once $\alpha_{s}$ is adjusted to reproduce $R_{A A}$ in central collisions.
\end{abstract}

Keywords: Relativistic heavy-ion collisions, parton energy loss, momentum anisotropy, elliptic flow

\section{Introduction}

Understanding parton energy loss in ultrarelativistic heavy-ion reactions has been the focus of considerable recent theoretical effort. A variety of phenomenological approaches (e.g., [1, 2, 3]) formulate the problem in terms of a local energy loss rate $d E / d L=-f(E(L), T(L), L)$ along the Eikonal parton trajectory, given by the local temperature, position, and parton energy. In the small-coupling regime, more rigorous treatment is possible based on perturbative QCD [4, 5, 6]. This includes quantum interference effects and also fluctuations, namely, energy loss along a given jet trajectory becomes a stochastic variable that is in general a function of the scattering and emission history of the jet.

A critical step in computing heavy-ion observables from any energy loss model is spatial and temporal averaging over the bulk medium formed in the collision. We employ here the Gyulassy-Levai-Vitev (GLV) framework[6] in which a high-energy parton loses energy through gluon radiation induced by interactions with static Yukawa scatterers in the medium. It is natural to combine this approach with parton transport for the bulk evolution, such as Molnar's Parton Cascade[7] (MPC), because it directly provides scattering center information.

Our approach is similar to recent work by Buzzatti and Gyulassy[8], but with a few key differences. Unlike [8], at present we only focus on light partons, and do not include multiple gluon radiation, elastic energy loss, or energy loss fluctuations due to variations in radiated gluon momentum. However, we do include realistic 3D medium evolution with both longitudinal and transverse expansion, which turns out to influence energy loss and, especially, elliptic flow.

\section{Radiative energy loss and medium averaging}

We consider here the leading $n=1$ (single scattering) term in the GLV opacity expansion of the radiated gluon spectrum [6]

$$
x \frac{d N^{(1)}}{d x d^{2} \mathbf{k}}=\frac{C_{R} \alpha_{s}}{\pi^{2}} \chi \int d^{2} \mathbf{q} \frac{\mu^{2}(z)}{\pi\left(\mathbf{q}^{2}+\mu^{2}(z)\right)^{2}} \frac{\mathbf{k q}}{\mathbf{k}^{2}(\mathbf{k}-\mathbf{q})^{2}}\left[1-\cos \left(\frac{(\mathbf{k}-\mathbf{q})^{2} z}{2 x E}\right)\right]
$$



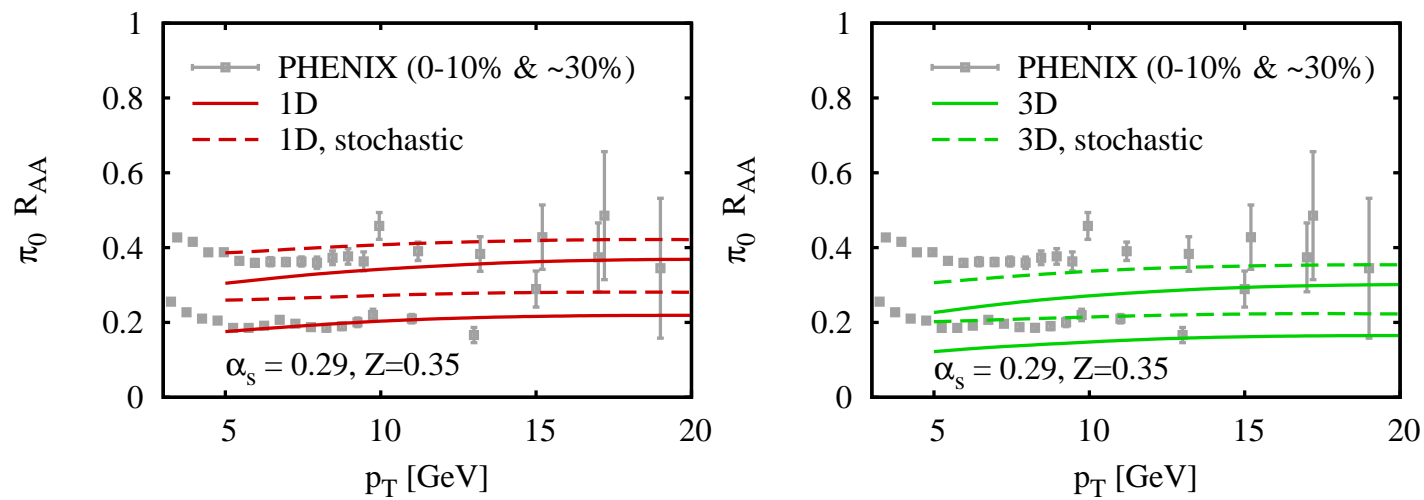

Figure 1. Neutral pion suppression factor $R_{A A}$ at midrapidity in central and mid-central Au+Au at $\sqrt{s_{N N}}=200 \mathrm{GeV}$ from calculations with GLV energy loss. Left: results for a medium undergoing longitudinal Bjorken expansion only. Right: results for realistic medium evolution with both longitudinal and transverse expansion (see text for details). In both cases, two scenarios are considered, one based on the average energy loss along the jet pass (solid), and one utilizing stochastic energy loss $\Delta E(z)$ (dashed). Data from PHENIX (boxes) are also shown[12] to guide the eye.

where the original hard scattering is at $z=0, \mu(z)$ is the local Debye screening mass, $\sigma=9 \pi \alpha_{s}^{2} /\left(2 \mu^{2}\right)$ is the (screened) total $g g \rightarrow g g$ scattering cross section, and $\chi=\int d z \rho, \sigma$ is the opacity. We integrated this spectrum numerically with kinematic bounds $k<x E, q<\sqrt{6 E T}$, and $x E \geq \mu$ to obtain a momentum-averaged energy $\operatorname{loss} \Delta E^{(1)}(z)=$ $\int d x d^{2} \mathbf{k} E x\left(d N^{(1)} / d x d^{2} \mathbf{k}\right)$ for fixed $z$, i.e., retained energy loss fluctuations due to variations in $z$ only. The probability for the scattering to occur at $z$ is $p(z)=\rho(z) \sigma(z) / \chi$, so the fully averaged energy loss is $\Delta E^{(1)}=\int d z p(z) \Delta E^{(1)}(z)$.
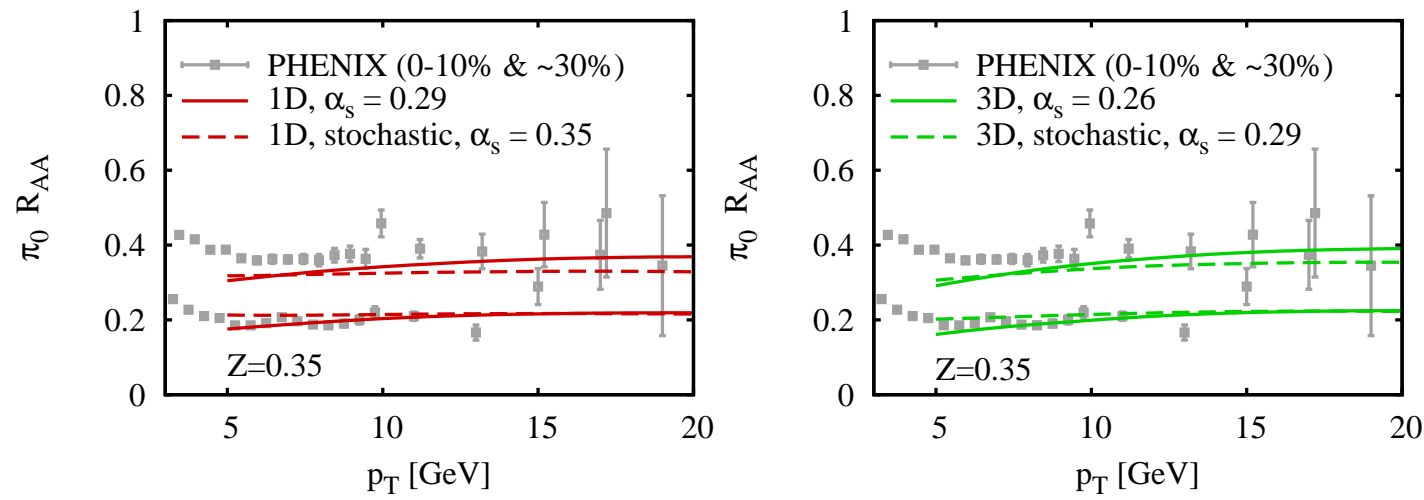

Figure 2. Same as Fig. 1 1 but with a slight tuning of $\alpha_{s}$ in each of the four scenarios to match the data above $p_{T} \gtrsim 8 \mathrm{GeV}$ in central collisions. After the tuning, all scenarios give practically the same result.

As customary, in non-static media we reinterpret $\rho(z)$ in the GLV formula as $\rho\left(z, t=t_{0}+z\right)$ along the parton trajectory. The density evolution was obtained from the parton transport MPC, employing $2 \rightarrow 2$ interactions for massless gluons. The scattering rate was adjusted to generate substantial $v_{2}\left(p_{T} \approx 3 \mathrm{GeV}\right) \sim 0.25$ in collisions with $b=8 \mathrm{fm}$ impact parameter, and we set growing $\sigma_{g g \rightarrow g g} \propto \tau^{2 / 3}$ to keep the shear viscosity to entropy ratio approximately constant[9]. Initial conditions for $\mathrm{Au}+\mathrm{Au}$ at $\sqrt{s_{N N}}=200 \mathrm{GeV}$ and $\mathrm{Pb}+\mathrm{Pb}$ at $\sqrt{s_{N N}}=2.76 \mathrm{TeV}$ were based on diffuse Woods-Saxon nuclei. For the transverse density, binary collision profiles were used, while the impact parameter dependence of rapidity densities $d N(b) / d y$ was proportional to $N_{\text {part }}$ with $d N(0) / d y=1100(\mathrm{Au}+\mathrm{Au})$ and $2400(\mathrm{~Pb}+\mathrm{Pb})$ to match the observed charged particle yields. Because we are only interested in observables at midrapidity, we set up boost invariant conditions in the coordinate rapidity window $|\eta|<5$, with formation time $\tau_{0}=0.6 \mathrm{fm}$. 

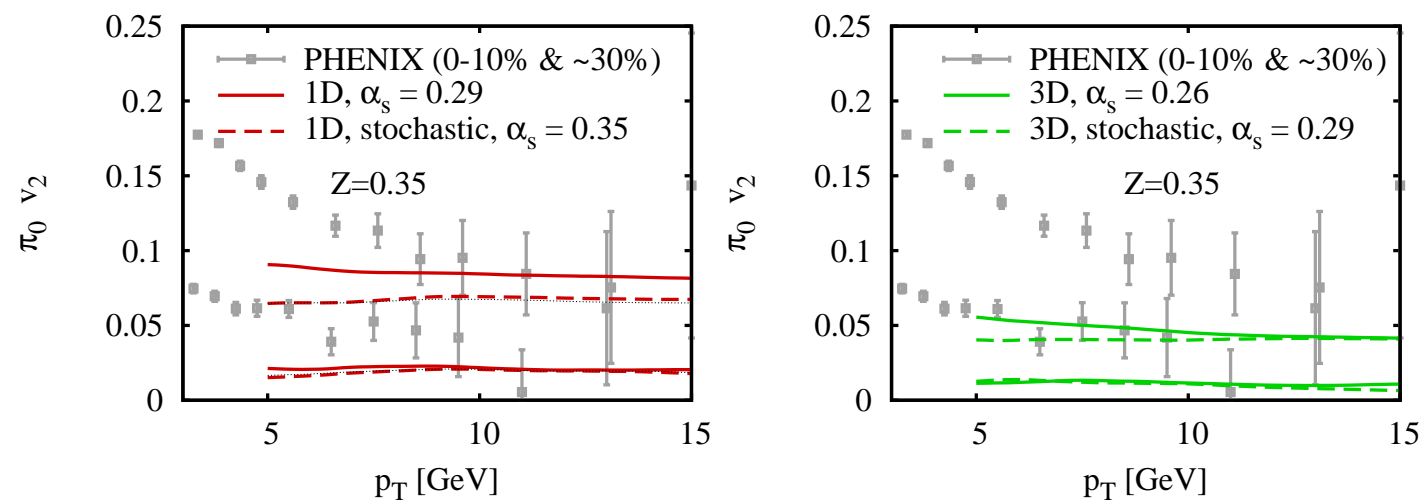

Figure 3. Differential $\pi_{0}$ elliptic flow $v_{2}\left(p_{T}\right)$ at midrapidity in central and mid-central Au+Au at $\sqrt{s_{N N}}=200 \mathrm{GeV}$ from calculations with GLV energy loss. The value of $\alpha_{s}$ was tuned to reproduce $R_{A A}$ in central collisions (cf. Fig. 2). Left: results for a medium undergoing longitudinal Bjorken expansion only. Right: results for realistic medium evolution with both longitidunal and transverse expansion (see text for details). In both cases, two scenarios are considered, one is based on the average energy loss along the jet pass (solid), and one utilizes stochastic energy loss $\Delta E(z)$ (dashed). With realistic 3D medium evolution we find a striking 40-50\% reduction in elliptic flow compared to the case of simplified 1D dynamics. Data from PHENIX (boxes) are also shown[13] to guide the eye.

Using tabulated densities from the transport, we set the local temperature assuming a massless gas of gluons $\rho \approx 2 T^{3}$ and the Debye mass via $\mu=g T \approx 2 T$. At early times $\tau<\tau_{0}$ we assume linear density build-up[8] $\rho=\tau \rho\left(\vec{x}_{T}, \tau_{0}\right) / \tau_{0}$. We roughly account for additional energy loss off dynamical (recoiling) scattering centers[10] $\left[\left(q^{2}+\mu^{2}\right)^{2} \rightarrow q^{2}\left(q^{2}+\mu^{2}\right)\right.$ in [1] $]$ and elastic scattering[11] via rescaling our opacities $\chi \rightarrow \chi / Z$ with $Z=0.35$. Initial jet momentum distributions in $\mathrm{p}+\mathrm{p}, \mathrm{Au}+\mathrm{Au}$ and $\mathrm{Pb}+\mathrm{Pb}$ were computed from leading-order $(\mathrm{LO})$ perturbative $\mathrm{QCD}$ with one-loop running coupling $\alpha_{s}\left(Q^{2}\right)$, using CTEQ5L parton distribution function parameterizations with $Q^{2}=p_{T, p a r t o n}^{2}$. Nuclear effects such as shadowing were ignored but isospin (proton-neutron difference) was included. After energy loss, jets were fragmented independently using LO BKK95 fragmentation function parameterizations with scale factor $Q^{2}=p_{T, \text { hadron }}^{2}$ and we assumed $\pi_{0}=\left(\pi^{+}+\pi^{-}\right) / 2$ for the neutral pion yield. This procedure reproduces high- $p_{T} \pi_{0}$ and charged particle spectra in $\mathrm{p}+\mathrm{p}$ at RHIC and LHC with modest $K_{N L O} \approx 2.5$ to account for higher-order contributions.

Below we focus on two basic high- $p_{T}$ observables for neutral pions at midrapidity, the nuclear suppression factor $R_{A A}$ and the momentum anisotropy (elliptic flow) $v_{2}=\langle\cos 2 \phi\rangle_{p_{T}}$. Only energy loss was considered, i.e., contributions by the radiated gluons to the final spectrum and feedback on the bulk medium due to the jet were ignored.

\section{Main results}

We considered four scenarios based on i) whether the medium is only undergoing Bjorken expansion ("1D" as in [8]) or transverse expansion as well (“3D”); and ii) whether average energy loss is used or the stochastic $\Delta E(z)$. Figure 1 shows our results for $R_{A A}$ at RHIC, for the same $\alpha_{s}=0.29$. In the stochastic case energy loss effects are noticeably weaker, which is natural for convex parton spectra ("curving up" at high $p_{T}$ ). We also find that realistic transverse expansion significantly enhances jet quenching, which is a generic GLV feature coming from the interference term in (1). Scatterings at large $z$ induce larger energy loss, and with a transversely expanding density profile there is higher chance to scatter further away from the production point than in the transversely static case.

Unfortunately, without precise control over $\alpha_{s}, R_{A A}$ alone cannot differentiate between these four scenarios. As shown in Fig. 2, after a slight tuning of $\alpha_{s}$ to reproduce the suppression in central collisions, differences in $R_{A A}$ largely disappear. On the other hand, striking difference in $v_{2}$ remains between 1D and 3D evolution at both RHIC and LHC energies, as shown in Figs. 3 and 4 The strong 40-50\% reduction of $v_{2}$ at high- $p_{T}$ with realistic transverse expansion is another generic consequence of interference in GLV. Scattering points that lead to most energy loss are biased to occur away from the production point and so later in time, by when the expansion makes the system more cylindrical, reducing the spatial azimuthal asymmetry that drives elliptic flow. We expect that this strong effect will be manifest in more full-fledged GLV calculations as well, such as [8]. 

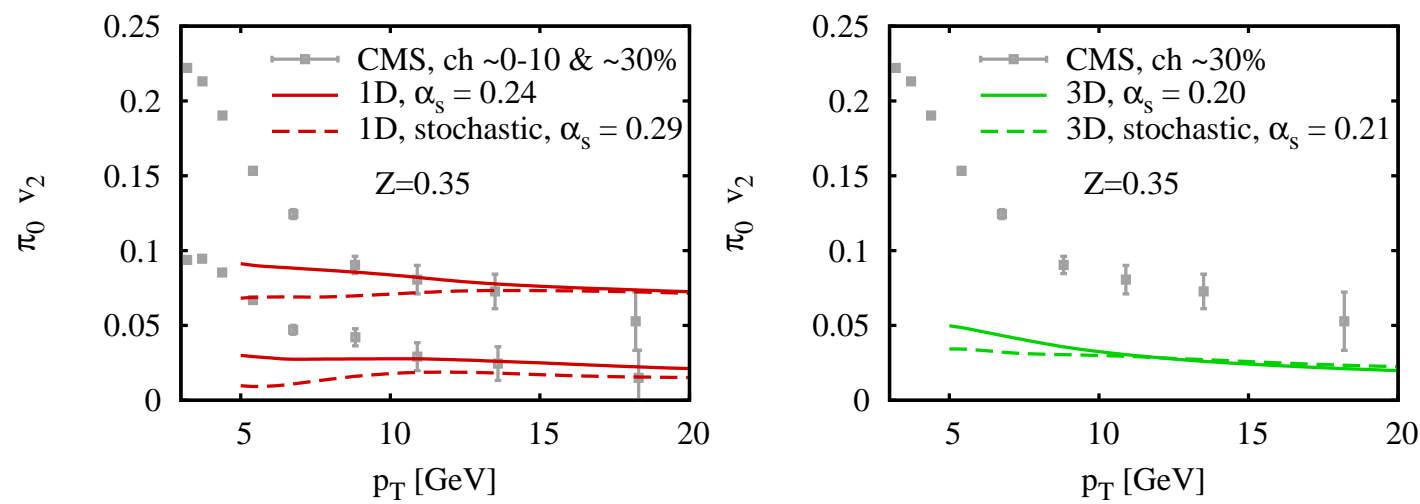

Figure 4. Same as Fig. 3 but for $\mathrm{Pb}+\mathrm{Pb}$ at $\sqrt{s_{N N}}=2.76 \mathrm{TeV}$ and with $\alpha_{s}$ tuned to reproduce $R_{A A} \approx 0.18$ at $p_{T}=6 \mathrm{GeV}$ in central collisions. With realistic 3D medium evolution (right) we only calculated $v_{2}$ for non-central collisions. Transverse expansion reduces $v_{2}$ dramatically at LHC energies as well. Charged particle $v_{2}\left(p_{T}\right)$ data from CMS (boxes) are also shown[14] to guide the eye.

At the conference we also presented results based on scattering center ensembles from the transport (not just density information) but due to page limitations these will be written up elsewhere.

\section{Conclusions}

We investigated GLV energy loss using bulk medium evolution data from the covariant transport MPC. We find that realistic transverse expansion strongly suppresses elliptic flow at high $p_{T}$ compared to calculations with transversely "frozen" profiles (as in [8]). We argue that this is a generic feature of GLV energy loss, raising the question whether GLV produces too little elliptic flow at high $p_{T}$.

Transverse expansion also enhances the high- $p_{T}$ suppression, while fluctuations in energy loss with the location of scattering centers reduce energy loss effects. However, unlike for $v_{2}$, these effects nearly disappear once calculations are adjusted to reproduce $R_{A A}$ in central collisions.

Acknowledgements: we thank A. Buzzatti and I. Vitev for stimulating discussions. This work was supported by the US DOE under grant DE-PS02-09ER41665. Also, D.S. was partially supported by the JET Collaboration (DOE grant DE-AC02-05CH11231).

\section{References}

[1] A. Drees, H. Feng and J. Jia, Phys. Rev. C 71, 034909 (2005) |nucl-th/0310044|; J. Jia and R. Wei, Phys. Rev. C 82, 024902 (2010) arXiv:1005.0645[nucl-th]].

[2] J. Liao and E. Shuryak, Phys. Rev. Lett. 102, 202302 (2009) arXiv:0810.4116 [nucl-th]].

[3] B. Betz and M. Gyulassy, Phys. Rev. C 86, 024903 (2012) |arXiv:1201.0281 [nucl-th]].

[4] R. Baier et al., Nucl. Phys. B 483 (1997) 291;

[5] U. A. Wiedemann, Nucl. Phys. A 690 (2001); C. A. Salgado and U. A. Wiedemann, Phys. Rev. D 68, 014008 (2003)

[6] M. Gyulassy, P. Levai and I. Vitev, Nucl. Phys. B 571 (2000) 197; M. Gyulassy, P. Levai and I. Vitev, Nucl. Phys. B 594, 371 (2001) nucl-th/0006010].

[7] D. Molnar and M. Gyulassy, Phys. Rev. C 62, 054907 (2000); D. Molnar, MPC 1.8.11. This transport code is available at http://karman.physics.purdue.edu/OSCAR

[8] A. Buzzatti and M. Gyulassy, Phys. Rev. Lett. 108, 022301 (2012) |arXiv:1106.3061 [hep-ph]].

[9] D. Molnar, arXiv:0806.0026 [nucl-th].

[10] M. Djordjevic and U. Heinz, Phys. Rev. C 77, 024905 (2008) |arXiv:0705.3439 [nucl-th]].

[11] S. Wicks, W. Horowitz, M. Djordjevic and M. Gyulassy, Nucl. Phys. A 783, 493 (2007) |nucl-th/0701063|.

[12] A. Adare et al. [PHENIX Collaboration], Phys. Rev. Lett. 101, 232301 (2008) [arXiv:0801.4020 [nucl-ex]].

[13] A. Adare et al. [PHENIX Collaboration], Phys. Rev. Lett. 105, 142301 (2010) arXiv:1006.3740 [nucl-ex]].

[14] S. Chatrchyan et al. [CMS Collaboration], arXiv:1204.1409 [nucl-ex]. 